\title{
Phase Transition in Quantum Gravity
}

\author{
Viqar Husain and Sebastian Jaimungal \\ Department of Physics and Astronomy, \\ University of British Columbia, \\ 6224 Agricultural Road, Vancouver, BC V6T 1Z1, Canada
}

\begin{abstract}
A fundamental problem with attempting to quantize general relativity is its perturbative non-renormalizability. However, this fact does not rule out the possibility that non-perturbative effects can be computed, at least in some approximation. We outline a quantum field theory calculation, based on general relativity as the classical theory, which implies a phase transition in quantum gravity. The order parameters are composite fields derived from spacetime metric functions. These are massless below a critical energy scale and become massive above it. There is a corresponding breaking of classical symmetry.
\end{abstract}

A quantum theory of gravity should have the property that its long distance regime is classical general relativity (GR). On the other hand, the only criteria for the short distance regime, in the absence of experimental guides, is self-consistency and finiteness. At first sight this appears to bestow a large degree of freedom on attempts at formulating a theory of quantum gravity. However, as is well known, there have been many unsuccessful attempts over the years, with partial successes coming mainly from string theory [1], and the loop quantum gravity program [2] .

From a conventional standpoint, a first question might be what classical theory is to be quantized to obtain quantum gravity. An obvious starting point, classical GR, leads to the non-renormalizability problem as far as perturbative quantum field theory (QFT) approaches are concerned. This problem in itself, however, does not imply that GR cannot be quantized. For example, the quantum theories of certain mini-superspace sectors of GR are known. Unfortunately, since these sectors correspond to quantum mechanical systems, they do not reveal much about what might be interesting properties of quantum gravity. Indeed, it may be argued that quantum mechanical reductions can reveal nothing significant about the full underlying quantum theory of gravity.

A next attempt at a starting point, short of full GR or supergravity, might be midi-superspace models - reductions of GR which are still field theories. One such reduction is obtained by imposing two Killing field symmetries. This reduction has been extensively studied with a view to quantization [3]. The most recent work is a claim that this dimensionally reduced theory can be completely quantized by 
finding a representation of the complete classical observable algebra [4]. Although apparently complete mathematically, this quantization has so far given little physical insight into the underlying quantum theory. It is therefore important to probe such models a bit further.

In this work we attempt to extract physical consequences from a path integral quantization of the two-Killing field reduction of GR. The model is a two dimensional field theory containing two local degrees of freedom which interact non-linearly. Non-perturbative considerations reveal that the quantum theory contains a phase transition. The order parameters for the transition are composite fields made from spacetime metric functions. We find that the composite fields are massless when the ultraviolet (UV) cutoff is below a critical energy scale, and are massive when it is above this scale.

Our starting point is the spacetime metric

$$
d s^{2}=e^{2 A}\left(-d t^{2}+d z^{2}\right)+g_{a b} d x^{a} d x^{b},
$$

where $A=A(t, z), x^{a}=\left(x^{1}, x^{2}\right)$, and the $2 \times 2$ metric

$$
g_{a b}=R\left(\begin{array}{cc}
\cosh W+\cos \Phi \sinh W & \sin \Phi \sinh W \\
\sin \Phi \sinh W & \cosh W-\cos \Phi \sinh W
\end{array}\right)
$$

is parameterized by the three functions $R(t, z), \Phi(t, z)$ and $W(t, z)$. The metric (1) has two commuting space-like Killing vector fields $\partial_{x^{1}}$ and $\partial_{x^{2}}$, whose orbits have the topology of $T^{2}$. The $t, z$ coordinates have ranges $0<t<\infty$ and $-\infty \leq z \leq \infty$. With these conventions, the metric is that of the Schmidt spacetime [5]. The vacuum Einstein equations in the gauge $R(t, \theta)=t$, give the two coupled twodimensional evolution equations

$$
\begin{gathered}
\ddot{W}+\frac{1}{t} \dot{W}-W^{\prime \prime}+\sinh W \cosh W\left(\Phi^{\prime 2}-\dot{\Phi}^{2}\right)=0, \\
\ddot{\Phi}+\frac{1}{t} \dot{\Phi}-\Phi^{\prime \prime}+2 \frac{\cosh W}{\sinh W}\left(\dot{\Phi} \dot{W}-\Phi^{\prime} W^{\prime}\right)=0,
\end{gathered}
$$

for $W(t, \theta)$ and $\Phi(t, \theta)$, and the two "constraint" equations

$$
\begin{aligned}
\dot{A}+\frac{1}{4 t}-\frac{t}{4}\left[\dot{W}^{2}+W^{\prime 2}+\sinh ^{2} W\left(\dot{\Phi}^{2}+\Phi^{\prime 2}\right)\right] & =0 \\
A^{\prime}-\frac{t}{2}\left(\dot{W} W^{\prime}+\sinh ^{2} W \dot{\Phi} \Phi^{\prime}\right) & =0 .
\end{aligned}
$$

The evolution equations involve $W(t, \theta)$ and $\Phi(t, \theta)$ and, given a solution to these equations, $A(t, \theta)$ is obtained by integration of the constraint equations (4-5).

The evolution equations (2-3) may be derived from a two-dimensional $\sigma$-modellike action

$$
S_{2}(W, \Phi)=\frac{1}{2} \int d t d \theta t \sqrt{-\eta} \eta^{a b} G_{A B}(Y) \partial_{a} Y^{A} \partial_{b} Y^{B}
$$


where $Y^{1}=W, Y^{2}=\Phi, \eta^{a b}=\operatorname{diag}(-,+), a, b, \cdots=t, z$, and $G_{A B}(Y) d Y^{A} d Y^{B}=$ $d W^{2}+\sinh ^{2} W d \Phi^{2}$ is the unit hyperboloid metric. The $t$ factor in the integrand cannot be absorbed by a rescaling of fields, or by introducing a curved two-dimensional metric. However this can be done if the model is embedded in three dimensions (see below).

Equations (2-3) can also be derived from the standard three-dimensional $S L(2, \mathbb{R})$ non-linear $\sigma$-model [6],

$$
S_{3}\left[X^{i}, \lambda, \mu^{i}\right]=\int_{M} d t d x d z \sqrt{-\eta}\left\{\eta^{a b} g_{i j} \partial_{a} X^{i} \partial_{b} X^{j}+\lambda\left(g_{i j} X^{i} X^{j}+1\right)+\mu^{i} g_{i j} D X^{j}\right\}
$$

where $g_{i j}=\operatorname{diag}(+,+,-), \eta_{a b} d x^{a} d x^{b}=-d t^{2}+d x^{2}+d z^{2}, \lambda(t, x, z)$ and $\mu^{i}(t, x, z)$ are Lagrange multiplier fields, $D=t \partial_{x}+x \partial_{t}$, and $i=1, \cdots, 3$. The Lagrange multiplier $\mu^{i}$ enforces the appropriate reduction to two dimensions, and the multiplier $\lambda$ enforces the constraints which give $X^{i}=X^{i}(W, \Phi)$.

For quantization there is the option of using the two or the three-dimensional action given above. Using the latter allows inclusion of quantum fluctuations in all three dimensions, whereas the former restricts these to two dimensions. Thus we expect on intuitive grounds that the quantum theories obtained via these two routes will not be the same. Because of the richer range for quantum fluctuations and the standard $\sigma$-model approach it allows, we use the three-dimensional action $S_{3}$.

A quantum theory may be defined by the path integral

$$
Z=\int[d X][d \lambda][d \mu] \mathrm{e}^{-i M_{P} S_{3}\left[X^{i}, \lambda, \mu^{i}\right]}
$$

where $M_{P}$ is the Planck mass. In this path integral, it is possible to perform the Gaussian integral over all the dynamical fields $X^{i}$. This leads to the quantum effective action for the fields $\lambda$ and $\mu^{i}$.

The saddle point evaluation of the remaining path integral, in its regime of validity, gives a first non-perturbative approximation for the quantum dynamics of $X^{i}$. This requires solutions of the Euler-Lagrange (EL) equations for $\lambda$ and $\mu^{i}$ derived from the quantum effective action. It is here that we find evidence of a phase transition.

The quantum effective action obtained after integrating over the fields $X^{i}$ is

$$
S_{3}^{e f f}=-\frac{3}{2} \operatorname{Tr} \ln (\square+\lambda)-i M_{p} \int d t d x d z\left[\lambda-\frac{1}{4} D \mu^{i}(\square+\lambda)^{-1} D \mu^{i}\right],
$$

where $\square=-\eta^{a b} \partial_{a} \partial_{b}$ is the three-dimensional flat space Laplacian. The EL equations for $\lambda$ and $\mu^{i}$ are well defined only in the presence of an UV cutoff, $\Lambda$, due to a trace term which appears in one of the equations. At this stage therefore, a second energy scale, $\Lambda$, enters our quantum model; all solutions of the EL equations depend on the cutoff. The simplest solutions are

$$
\lambda=\lambda^{*}=\text { const. } \quad \text { and } \quad \mu^{i}=\mu^{i *}=\text { const.. }
$$




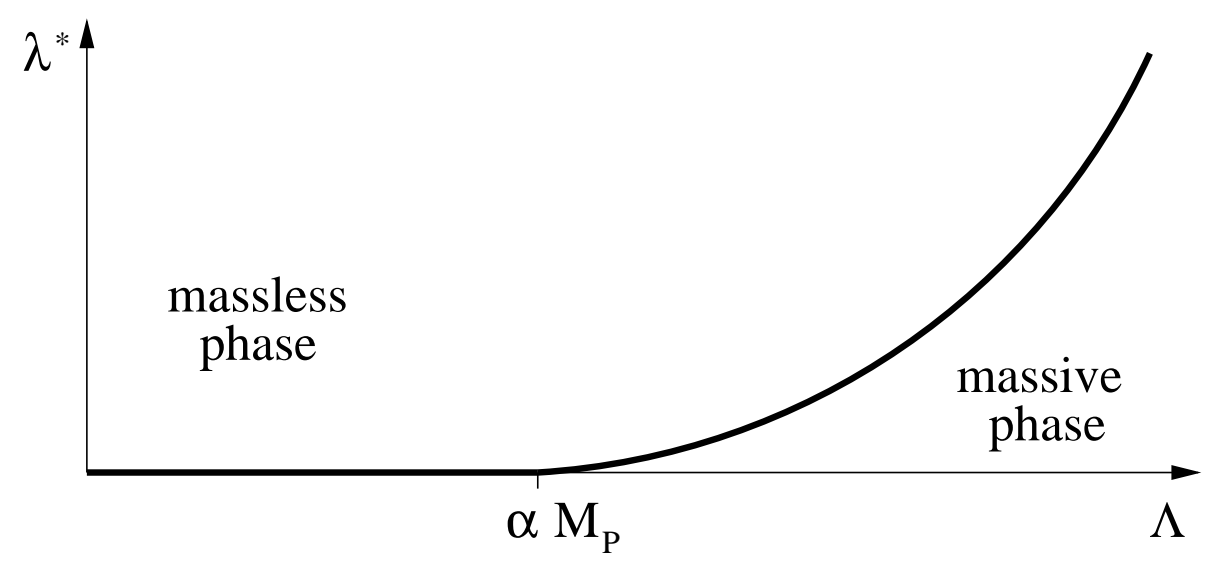

FIGURE 1. Spontaneous symmetry breaking occurs for $\Lambda<\alpha M_{P}$ and gravitational excitations become massless.

These particular solutions exist only if $\Lambda \geq \alpha M_{P}$ [7], where $\alpha$ is dimensionless constant of order unity.

What do these solutions imply for the phase of the theory? In the saddle point approximation, the two-point function is

$$
\left\langle X^{i}(x) X^{j}(y)\right\rangle \sim g^{i j} \int^{\Lambda} \frac{d^{3} k}{(2 \pi)^{3}} \frac{e^{i k(x-y)}}{k^{2}-\lambda^{*}},
$$

This simple result demonstrates that the $X^{i}$ fields are massive with mass $\lambda^{*}$ on scales $\Lambda>\alpha M_{P}$, and so we can speak of a "massive phase" in this regime. A careful treatment reveals that

$$
\lambda^{*}\left(\Lambda, M_{P}\right)=\text { const. } \frac{\Lambda^{4}}{\alpha^{2}}\left(\frac{1}{M_{P}}-\frac{\alpha}{\Lambda}\right)^{2},
$$

so that the mass is zero at $\Lambda=\alpha M_{P}$.

It is surprising that integrating out all the $X^{i}$ fields in eqn. (7) leads to these solutions of the effective action only if $\Lambda \geq \alpha M_{P}$. Is it possible to extend this solution to the region $\Lambda<\alpha M_{P}$ ? Indeed, it is. One must go back to the original path integral and assume that one of the $X^{i}$ fields is a constant, that is, it has a non-zero vacuum expectation value (vev). This modifies the EL equations for $\lambda$ and $\mu^{i}$, such that now $\lambda=0$ and $\mu^{i}=\mu^{i *}=$ constant are indeed solutions, (while the vev is found to depend on $\Lambda$ and $\left.M_{P}\right)$. Thus, in the $\Lambda<\alpha M_{P}$ parameter regime, we are in a "massless phase," and there is spontaneous breaking of the $S L(2, \mathbb{R})$ symmetry (due to the direction picked out by the constant component of the $X^{i}$ field). These results are summarized in Figure 1.

The steps we have outlined are standard for exploring the possibility of phase transitions in QFT. What is surprising, as we have seen, is that an analogous treatment applies to the path integral (7) for reduced Einstein gravity. This appears to be the first concrete indication from GR itself that a phase transition occurs at very short distances. 
What do these results imply for quantum gravity? Perhaps the main insight is that gravitational excitations are massive above the Planck energy scale, and massless, with spontaneous symmetry breaking, below this scale. The symmetry breaking below $M_{P}$ in this reduction of GR is relatively slight - it is a breaking of global $S L(2, \mathbb{R})$. Nevertheless, it is significant in that it occurs at all.

It is important to emphasize that this phenomena is essentially due to the two non-linearly interacting degrees of freedom in this model; it is missed if only one field is present. The implication of our result for full quantum gravity is the possibility that the graviton is a massless Goldstone boson associated with spontaneous symmetry breaking at the Planck scale.

Several questions remain for further research: Is the dimensionally reduced model considered here renormalizable? If so what is the $\beta$-function and its fixed points (if any)? What is the spacetime metric at scales below the Planck length?

With respect to the first question, it is well known that the regular $\sigma$-model in three dimensions is renormalizable. Therefore it is clear that this must be the case also for the present model, even with the dimensional reduction constraint it contains. Surprisingly, however, this result is not straightforward to establish if the constraint is first solved classically and an action in two dimensions is used as the starting point. This is because the resulting $\sigma$-model like action has an explicit time factor in the integrand, which results in the kinetic operator not being Sturm-Lioville. This crucially affects the standard evaluation of the Gaussian integral. Thus, establishing renormalizability and calculating the $\beta$-function is straightforward from the three-dimensional perspective, but not from the twodimensional one. Indeed the model may not even be renormalizable from the latter perspective, where the $\sigma$ model constraint is explicitly time dependent. If so, this would be yet another indication of the important differences between dimensionally reducing classically and then quantizing, versus quantizing by including the constraint quantum mechanically as we have done here.

\section{REFERENCES}

1. M. Green. J. Schwarz, and E. Witten, Superstring Theory (University of Cambridge Press (New York,1990)); J. Polchinski, String Theory: Superstring Theory and Beyond, (Cambridge University Press (New York, 1998)).

2. For a recent review see: C. Rovelli, "Loop Quantum Gravity," gr-qc/9710008.

3. An incomplete selection of papers is: K. Kuchař, Phys. Rev. D4, 955 (1971); B. Berger, Ann. Phys. 83, 458 (1974); V. Husain, Class. Quantum Grav. 41587 (1987); D. Korotkin and H. Nicolai, Phys. Rev. Lett. 74, 1272 (1995); G. Mena-Marugan Phys. Rev. D56, 908 (1997).

4. D. Korotkin and M. Samptleben, Phys. Rev. Lett. 80, 14 (1998).

5. B. G. Schmidt, Class. Quantum Grav., 13 (1996) 2811.

6. A. Ashtekar and V. Husain, Int. J. Mod. Phys. D7, 549 (1998).

7. A. M. Polyakov, Gauge Fields and Strings, (Harwood, NY, (1988)). 